\title{
A novel endoglin mutation in hereditary hemorrhagic telangiectasia type 1: A case report
}

\author{
YANJUN LU ${ }^{1}$, YAOWU ZHU ${ }^{1}$, LILI SHI ${ }^{2}$, HONGTAO ZHEN ${ }^{2}$, ZIYONG SUN $^{1}$ and LIMING CHENG ${ }^{1}$ \\ Departments of ${ }^{1}$ Laboratory Medicine and ${ }^{2}$ Otolaryngology-Head and Neck Surgery, Tongji Hospital, \\ Tongji Medical College, Huazhong University of Science and Technology, Wuhan, Hubei 430030, P.R. China
}

Received May 22, 2014; Accepted February 10, 2015

DOI: $10.3892 / \mathrm{mmr} .2015 .3442$

\begin{abstract}
Hereditary hemorrhagic telangiectasia (HHT) is an autosomal dominant disorder characterized by aberrant vascular development. Mutations in two genes, endoglin (ENG) and activin receptor-like kinase 1 (ACVRL1) are associated with HHT. The present case study revealed the molecular diagnosis in a family exhibiting the clinical features of HHT disease. The coding exon and flanking intronic regions of the ENG and ACVRL1 genes were sequenced and a novel mutation in exon 10 of ENG was observed in the family. The mutation (c.1426C $>$ T) in exon 10 of the ENG gene caused a G476X mutation, which results in a premature stop codon and a truncated ENG protein. This finding demonstrated a novel mutation in the ENG gene in a Chinese family, which suggested that a truncated ENG protein may cause HHT. The present study established a genetic test to confirm the clinical diagnosis in individuals and provide an opportunity for early detection and management of the disease.
\end{abstract}

\section{Introduction}

Hereditary hemorrhagic telangiectasia (HHT), also termed Rendu-Osler-Weber syndrome, is an autosomal dominant disease characterized by telangiectases and arteriovenous malformations (AVMs) of the skin, mucosa and viscera, with direct arteriovenous connections lacking capillary beds. One of the predominant manifestations of HHT is nose bleeding or epistaxis (1). Clinical diagnosis of HHT is based on the family history, recurrent nosebleeds, mucocutaneous telangiectasis, and AVMs in the brain, lung, liver and gastrointestinal tract (2).

Genetic studies have demonstrated that heterozygous mutations in endoglin (ENG) or activin receptor-like kinase 1

Correspondence to: Dr Liming Cheng, Department of Laboratory Medicine, Tongji Hospital, Tongji Medical College, Huazhong University of Science and Technology, 1095 Jiefangdadao, Wuhan, Hubei 430030, P.R. China

E-mail: chengliming2002@163.com

Key words: hereditary hemorrhagic telangiectasia, endoglin, activin receptor-like kinase 1
(ACVRL1) cause HHT. The ENG gene is located on chromosome 9q34.1 and consists of 15 exons (exon 9 has two parts: 9a and 9b), which encode ENG, a type III transforming growth factor (TGF)- $\beta$ receptor. Mutations in the ENG gene may lead to type 1 HHT (3). Type 2 HHT is attributed to mutations in the ACVRL1 gene, which is located on chromosome 12q13. A total of 9/10 exons (exon 2-10) of the ACVRL1 gene code for the ACVRL1 protein, a type I TGF- $\beta$ receptor (4). These gene products are involved in TGF-associated angiogenesis.

Several mutations in the two genes have been identified in patients with HHT worldwide (5). However, few studies have been performed using Chinese individuals. In the present study, a family diagnosed with HHT was reported and the coding exons and flanking intronic regions of the ACVRL1 and ENG genes were sequenced. This revealed a novel mutation in the ENG gene. This finding identified a novel truncated ENG protein that causes HHT and a presymptomatic mutation carrier in the family, providing an opportunity for early detection and management of the disease.

\section{Case report}

A 38 year-old male patient was referred to the Department of Laboratory Medicine, Tongji Hospital (Hubei, China) with a history of epistaxis lasting $>20$ years. Systemic evaluation revealed minor skin telangiectasia. For several years, epistaxis was frequent and the patient learned to pack his own nose. In 2010, the patient was admitted to the Department of Laboratory Medicine, Tongji Hospital for anemia and frequent nose bleeding. In 2012, the patient was admitted again for epistaxis. A skull and brain magnetic resonance imaging/magnetic resonance angiography revealed no telangiectasia lesions. The patient was treated with traditional temporary relief for the nose bleeds. However, epipharyngoscope revealed multiple telangiectasia lesions throughout the nose (Fig. 1). The present case study was approved by the ethics committee of The Ethical Committee of Tongji Hospital, Tongji Medical College, Huazhong University of Science and Technology, Wuhan,China.

The patient's medical and family history was carefully investigated. The son of the proband ( 7 years old) exhibited frequent nosebleeds. The patient's father and uncle had a long history of epistaxis, and the eventual cause of mortality was cerebral hemorrhage. The patient's brother and cousin also had 

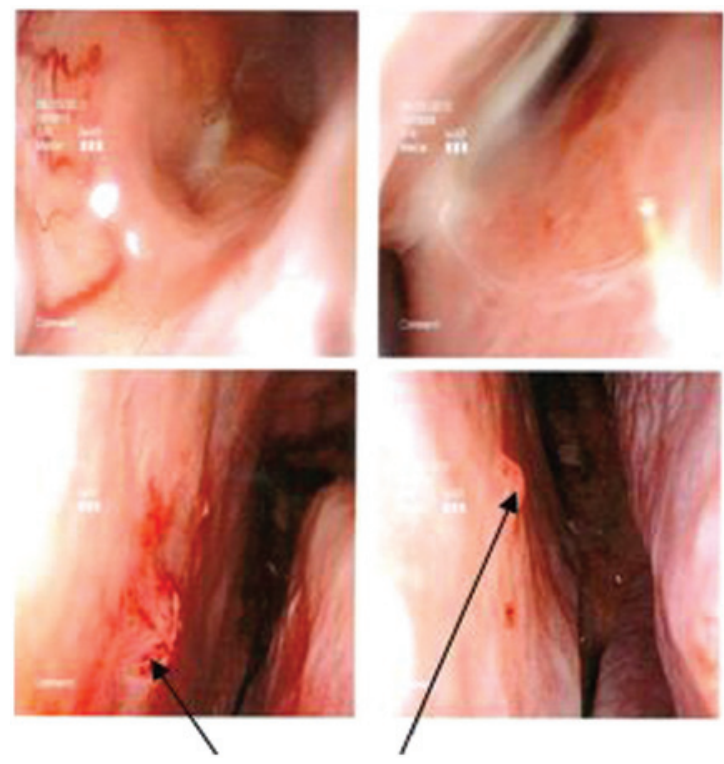

Figure 1. Epipharyngoscope revealed the lesions in patients with hereditary hemorrhagic telangiectasia type 1. The upper panel demonstrates the normal nasal cavity, the lower panel demonstrates the nasal septum of the patient with HHT. The arrows highlight the hemorrhagic foci.

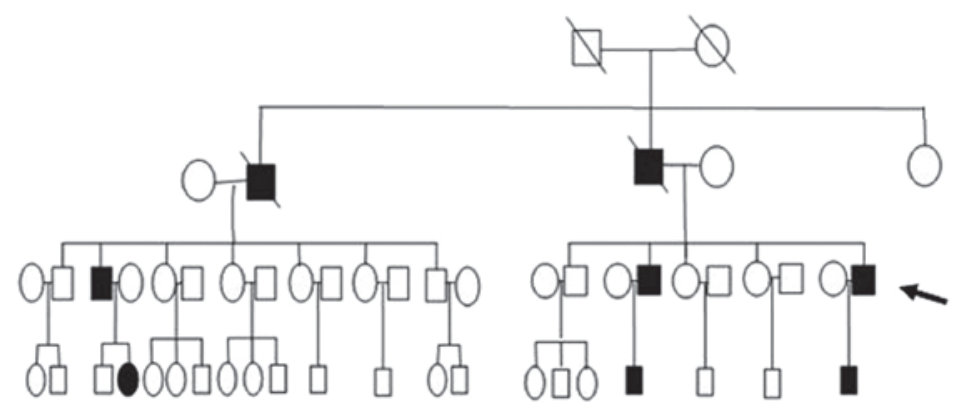

Figure 2. Pedigree of family with HHT1. There are eight individuals with HHT1, including the proband, and 42 healthy individuals in this family. HHT1, hemorrhagic telangiectasia type 1 .

A
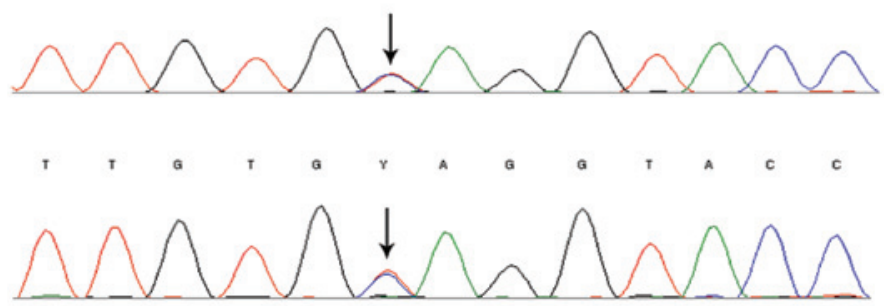

B

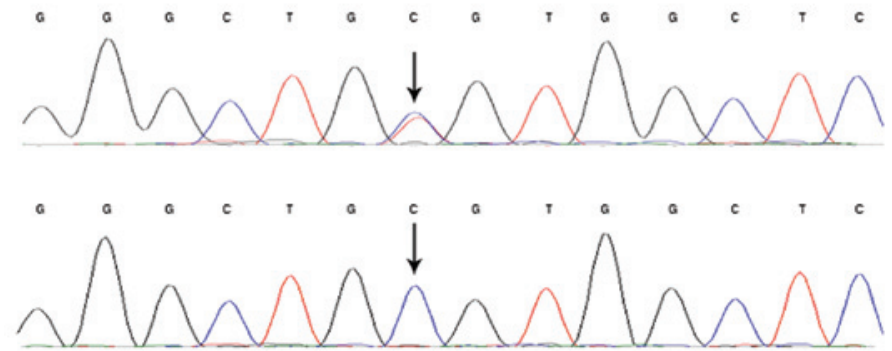

Figure 3. Clinical sequencing results from the two patients with hereditary hemorrhagictelangiectasia presented. (A) The proband (upper) and the son (lower) exhibited a heterozygous exon 10 of endoglin mutation (c.1426C >T; pz476X). (B) The proband (upper) exhibited a heterozygous synonymous mutation c.159C $>\mathrm{T}$ and the son (lower) exhibited no mutation in exon 2. 
a long history of epistaxis. The family history of epistaxis is shown in Fig. 2. The family history and recurrent epistaxis, requiring cautery, resulted in the patient being referred for HHT molecular testing. Informed consent for clinical genetic testing was obtained for all individuals tested. Genetic screening of the ENG and ACVRL1 genes was performed on all family members. Two mutations were detected in the ENG gene from the proband, one synonymous mutation $(159 \mathrm{C}>\mathrm{T}$ in exon 2) and a nonsense heterozygous mutation $(1426 \mathrm{C}>\mathrm{T}$ in exon 10). The nonsense mutation was also detected in the other family members with HHT and a presymptomatic mutation carrier (the son of the proband's brother; 2 years old). However, the synonymous mutation $159 \mathrm{C}>\mathrm{T}$ was only detected in the proband, which suggested that this was a novel mutation. The sequencing results are shown in Fig. 3.

\section{Discussion}

The present case study reported a family with clinically and genetically confirmed HHT. Genetic screening of two of the HHT-associated genes, ACVRL1 and ENG, in the Chinese family revealed a substitution mutation at codon 1426 on exon 10 of the ENG gene. This substitution mutation (C1426A) leads to CAG (Gln, G) being changed into TGA (termination codon, G476X). This was the first time, to the best of our knowledge, that this novel mutation was observed in a patient with HHT. It has been demonstrated that in ENG, $\sim 15 \%$ of sequence variants are nonsense, $38 \%$ are small deletions/duplications, $7 \%$ are large deletions/duplications, $12 \%$ are splice site mutations and $27 \%$ are missense mutations. It has been suggested that these mutant proteins may cause a dominant negative effect by disrupting normal endoglin function $(6,7)$.

ENG is a member of the type III TGF- $\beta$ family receptors and functions as a homodimer. Of all HHT1 cases, $~ 80 \%$ lead to premature stop codons and truncated endoglin proteins, and the resultant protein may act as a dominant negative allele by reducing the fraction of the protein produced from the remaining normal allele (8). It has been demonstrated that mutated endoglin proteins are expressed at low levels, mediated by nonsense mediated mRNA decay (9). Notably, patients with mutations in endoglin have significantly lower endoglin levels in peripheral blood monocytes compared with the controls (10). It has been hypothesized that the mutated proteins may be misfolded and unstable, and fail to reach the cell surface, therefore rendering them unable to form heterodimers with normal endoglin (6). This implies that haplo-insufficiency is the predominant underlying mechanism; however, individuals with HHT exhibit great variability in the age of disease onset, and clinical manifestations and severity, even within a family with the identical mutation. This suggested that other mechanisms may account for the development of the clinical symptoms.

HHT1 and HHT2 are clinically similar in presentation, however, vary in genotype-phenotype correlation studies. A previous study suggested that the clinical manifestations of mutations in ENG are more severe compared with those caused by mutations in ACVRL1 (11), with an earlier age of onset of epistaxis and the appearance of telangiectasias, and a higher incidence of pulmonary AVMs.

In conclusion, the molecular investigation of ACVRL1 and ENG is an important diagnostic tool for confirmation of the clinical diagnosis. In the present study, a novel mutation in ENG was revealed, which may contribute to the clinical phenotypes exhibited by this family. Furthermore, in the investigated family, all asymptomatic family members were screened and exhibited no mutation, with the exception of one individual. This suggested that genetic screening of asymptomatic family members may allow the detection of harmful forms of vascular lesions in advance, prior to complications arising. The patient was advised to maintain humid air and to apply lubricants, including saline, to prevent crusting over the nasal mucosa. Multi-slice CT and MRI were performed to determine whether there was the visceral hemorrhage.

\section{References}

1. Braverman IM, Keh A and Jacobson BS: Ultrastructure and threedimensional organization of the telangiectases of hereditary hemorrhagic telangectasia. J Invest Dermatol 95: 422-427, 1990.

2. Shovlin CL, Guttmacher AE, Buscarini E, Faughnan ME, Hyland RH, Westermann CJ, Kjeldsen AD and Plauchu H: Diagnostic criteria for hereditary hemorrhagic telangiectasia (Rendu-Osler-Weber syndrome). Am J Med Genet 91: 66-67, 2000.

3. McAllister KA, Grogg KM, Johnson DW, Gallione CJ, Baldwin MA, Jackson CE, Helmbold EA, Markel DS, McKinnon WC and Murrell J: Endoglin, a TGF-beta binding protein of endothelial cells, is the gene for hereditary haemorrhagic telangiectasia type 1. Nat Genet 8: 345-351, 1994.

4. Johnson DW, Berg JN, Baldwin MA, Gallione CJ, Marondel I, Yoon SJ, Stenzel TT, Speer M, Pericak-Vance MA, Diamond A, Guttmacher AE, Jackson CE, Attisano L, Kucherlapati R and Porteous ME, Marchuk DA: Mutations in the activin receptor-like kinase 1 gene in hereditary haemorrhagic telangiectasia type 2. Nat Genet 13: 189-195, 1996.

5. Bossler AD, Richards J, George C, Godmilow L and Ganguly A. Novel mutations in ENG and ACVRL1 identified in a series of 200 individuals undergoing clinical genetic testing for hereditary hemorrhagic telangiectasia (HHT): correlation of genotype with phenotype. Hum Mutat 27:667-675, 2006.

6. Abdalla SA and Letarte M: Hereditary haemorrhagic telangiectasia: current views on genetics and mechanisms of disease. J Med Genet 43: 97-110, 2006.

7. Prigoda NL, Savas S, Abdalla SA, Piovesan B, Rushlow D, Vandezande K, Zhang E, Ozcelik H, Gallie BL and Letarte M: Hereditary haemorrhagic telangiectasia: mutation detection, test sensitivity and novel mutations. J Med Genet 43: 722-728, 2006.

8. McAllister KA, Baldwin MA, Thukkani AK, Gallione CJ, Berg JN, Porteous ME, Guttmacher AE and Marchuk DA: Six novel mutations in the endoglin gene in hereditary hemorrhagic telangiectasia type 1 suggest a dominant-negative effect of receptor function. Hum Mol Genet 4: 1983-1985, 1995.

9. Frischmeyer PA and Dietz HC: Nonsense-mediated mRNA decay in health and disease. Hum Mol Genet 8: 1893-1900, 1999.

10. Pece-Barbara N, Cymerman U, Vera S, Marchuk DA and Letarte M. Expression analysis of four endoglin missense mutations suggests that haploinsufficiency is the predominant mechanism for hereditary hemorrhagic telangiectasia type 1 . Hum Mol Genet 8: 2171-2181, 1999.

11. Berg J, Porteous M, Reinhardt D, Gallione C, Holloway S, Umasunthar T, Lux A, McKinnon W, Marchuk D and Guttmacher A: Hereditary haemorrhagic telangiectasia: a questionnaire based study to delineate the different phenotypes caused by endoglin and ALK1 mutations. J Med Genet 40: 585-590, 2003. 